\title{
Reflets
}

Revue ontaroise d'intervention sociale et communautaire

\section{Quand le harcèlement se mêle au travail ou aux études...}

\section{Marie-Luce Garceau}

Volume 9, numéro 2, automne 2003

Travail et mieux-être

URI : https://id.erudit.org/iderudit/011091ar

DOI : https://doi.org/10.7202/011091ar

Aller au sommaire du numéro

Éditeur(s)

Reflets : Revue ontaroise d'intervention sociale et communautaire

ISSN

1203-4576 (imprimé)

1712-8498 (numérique)

Découvrir la revue

Citer cet article

Garceau, M.-L. (2003). Quand le harcèlement se mêle au travail ou aux études... Reflets, 9(2), 58-86. https://doi.org/10.7202/011091ar

\section{Résumé de l'article}

Le harcèlement sexiste et sexuel existe bel et bien en milieu postsecondaire dans les institutions du Nord de l'Ontario. L'ampleur du harcèlement sexiste et sexuel est élevée puisque près de sept femmes sur dix qui étudient ou qui travaillent dans ces milieux, disent en avoir été les victimes. Dans ces milieux, le harcèlement s'exerce principalement par l'instauration d'un climat sexiste et d'un climat empoisonné de travail et d'études entre pairs. L'auteure insiste, en conclusion, sur l'importance que l'on doit accorder au phénomène, tout particulièrement parce que les institutions d'éducation ont le devoir de soutenir les étudiantes comme leur personnel.
Tous droits réservés (C) Reflets : Revue ontaroise d'intervention sociale et communautaire, 2002
Ce document est protégé par la loi sur le droit d'auteur. L'utilisation des services d'Érudit (y compris la reproduction) est assujettie à sa politique d'utilisation que vous pouvez consulter en ligne.

https://apropos.erudit.org/fr/usagers/politique-dutilisation/ 


\title{
Quand le harcèlement se mêle au travail ou aux études...
}

\author{
Marie-Luce Garceau \\ Membre du Comité ad hoc sur la violence en milieu postsecondaire dans \\ le Nord de l'Ontario, Sudbury ${ }^{1}$
}

\section{Introduction}

Le harcèlement sexiste et sexuel en milieu de travail ou d'études est une sorte de guerre des nerfs, n'ayant rien à voir avec les petits accrocs de la vie professionnelle ou étudiante. Il s'agit de comportements, manifestement volontaires, d'une ou de plusieurs personnes, à l'endroit d'un autre individu ou d'un groupe: collègues, subalternes ou cadres hiérarchiques. Les formes des violences subies sont telles qu'elles peuvent dégrader les conditions de travail ou d'études, humilier, maltraiter, voire briser les femmes et les hommes qui les subissent.

Cela dit, c'est par l'entremise d'une recherche sur le harcèlement sexuel et sexiste en milieu postsecondaire en Ontario, que le Comité ad hoc sur la violence en milieu postsecondaire dans le Nord de l'Ontario s'est aperçu de l'importance que revêt ce phénomène dans les relations de travail et d'études ${ }^{2}$. L'objectif de l'article vise donc à mieux cerner cette situation particulière et à prendre conscience de l'ampleur du harcèlement sexiste et sexuel. Contrairement au rapport original du Comité ad hoc, qui visait à saisir le phénomène du harcèlement sexiste et sexuel en milieu postsecondaire du Nord de l'Ontario, cet article cherchera plutôt à présenter les données relatives au climat de travail. Mais d'abord posons le contexte. 


\section{Définitions du harcèlement sexiste et sexuel}

"La majorité des études indiquent que les femmes sont plus susceptibles d'être harcelées par des hommes que l'inverse...."
Le harcèlement sexiste et sexuel est un problème de société et les femmes en sont les principales victimes. Or, les deux qualificatifs associés au terme de harcèlement évoquent une même réalité: celle de rabaisser et d'humilier les femmes, de les remettre à leur place en leur rappelant les normes du système patriarcal. Ils légitiment les rapports sociaux de domination des hommes sur les femmes en leur imposant un statut préconçu d'inférieures ou de subordonnées. Il rappelle aux femmes leur appartenance au modèle social féminin. Il s'agit d'un continuum de comportements qui favorisent un rapport d'abus de pouvoir et une vision sexiste des femmes ${ }^{3}$.

La majorité des études indiquent que les femmes sont plus susceptibles d'être harcelées par des hommes que l'inverse, que le pouvoir masculin se fait particulièrement sentir en milieu de travail et que le harcèlement sexuel s'exerce aussi dans les milieux d'études ${ }^{4}$. La principale conséquence du harcèlement est une limitation d'une participation pleine et entière à la société. Il empêche les femmes de vivre sans crainte, limitant leur accès à l'égalité et à l'équité.

Les études sur le harcèlement sexiste et sexuel présentent diverses formes qui s'inscrivent dans un continuum d'actes ou de comportements allant de la blague sexiste désobligeante et du regard importun à l'agression sexuelle accompagnée de violence. Pour l'essentiel, il s'agit de formes de harcèlement: verbal (demandes claires ou sous-entendues, etc.); non verbal (affichage pornographique, notes ou regards humiliants, etc.) ou physique (attouchements jusqu'à l'agression sexuelle avec violence). Ce continuum comporte des différences de degré et d'intensité.

Le harcèlement sexiste et sexuel est souvent associé à des incidents répétés. Toutefois, pour la Commission canadienne des droits de la personne, on mentionne qu'il peut s'agir «d'une manifestation isolée ou d'incidents répétés» et que les «moyens utilisés peuvent varier et augmenter en fréquence ou en intensité». 
"...il est urgent que

les femmes qui

étudient ou qui

travaillent dans les

institutions

postsecondaires et francophones du Nord de l'Ontario n'aient plus à se sentir humiliées, abaissées, impuissantes ou blessées."
Finalement, le harcèlement sexuel peut s'exercer à l'endroit de subordonnés ou de collègues. Cela signifie que le harcèlement sexuel ne suppose pas nécessairement une relation hiérarchique entre la personne qui harcèle et celle qui en est l'objet (Commission canadienne 1998: 7). Au contraire, d'après Soares (2002), «il peut se développer verticalement dans la hiérarchie de l'organisation ou horizontalement (entre collègues d'un même niveau hiérarchique» (p. 8). À ces deux formes, Soares indique qu'il est «nécessaire d'ajouter un troisième type où la gestion est responsable du harcèlement par incompétence managériale ou par absence d'un style de leadership» (p. 8-9).

Il existe, encore aujourd'hui, une résistance à reconnaître le harcèlement sexiste et sexuel, comme il existe une tentation très forte à le banaliser, à le minimiser, voire à le taire. Pourtant, les femmes qui subissent les comportements harcelants en paient le prix, car le harcèlement sexiste et sexuel a de nombreuses conséquences. Certes, les femmes ne réagissent pas toutes de la même manière, mais plusieurs subissent des effets psychologiques, physiques ou économiques importants. Par conséquent, pour le Comité ad hoc qui a encadré la recherche, il est urgent que les femmes qui étudient ou qui travaillent dans les institutions postsecondaires et francophones du Nord de l'Ontario n'aient plus à se sentir humiliées, abaissées, impuissantes ou blessées. Selon le Comité, elles ne doivent plus avoir à subir le stress du harcèlement sexiste et sexuel; elles ne doivent plus à avoir peur des représailles. Si elles dénoncent, on ne doit pas mettre en cause leur crédibilité; si elles osent en parler, elles ne doivent plus se faire accuser de provocation; si elles portent plainte, elles ne doivent plus changer de cours ou aller travailler ailleurs. En effet, le harcèlement sexiste et sexuel instaure souvent un climat de travail ou d'études insupportable, invivable pour celles qui le subissent.

Dans la littérature, le terme harcèlement sexiste et sexuel fait l'objet de nombreux débats quant à sa définition et surtout, son interprétation. Afin de tenter de cerner cette notion, souvent considérée comme floue ou élastique, nous avons construit quelques catégories d'analyse qui ont permis d'étudier certaines facettes du phénomène. 


\section{Catégories d'analyse}

Les définitions du harcèlement sexiste et sexuel étant en place, aux fins de la présente étude, nous avons regroupé 20 comportements dans quatre catégories: climat sexiste, climat empoisonné, harcèlement sexuel et agression sexuelle.

\section{Climat sexiste}

On peut qualifier de harcèlement sexiste les comportements tels: déshabiller des yeux, poser des regards insistants ou déplacés, faire des remarques négatives sur l'apparence, faire des remarques ou avoir des comportements sexistes tels des blagues et des taquineries à caractère sexuel. Dans la présente enquête, nous parlerons de climat sexiste pour désigner ce genre de comportements insidieux. Ces comportements se réferent au sexisme ambiant et ciblent explicitement les femmes. Ce sont les situations les plus fréquentes, celles qui passent souvent inaperçues tellement elles font partie du quotidien. Pourtant, ces comportements sexistes gênent ou choquent les personnes qui en sont l'objet, laissent des traces, des blessures. Ces comportements créent un doute qui ébranle la confiance de la personne qui les subit. Ils jettent les bases de comportements toujours plus abaissants et dénigrants pour les femmes, car ils soulignent que les femmes sont considérées comme des objets et occupent une position inférieure.

\section{Climat empoisonné}

Il existe une seconde catégorie de comportements harcelants que nous avons regroupés sous le terme de climat empoisonné. Il s'agit des comportements liés aux relations interpersonnelles qui détruisent l'ambiance au travail ou aux études et qui nuisent à la productivité des personnes qui en sont les victimes. On songe ici à l'intimidation, aux humiliations, aux vexations, voire aux menaces de réprimandes ou de renvoi qui créent un environnement hostile et minent le climat de travail ou d'études. Le but de ces comportements est de harceler personnellement ou moralement, 
d'asservir, de diminuer la personne qui en est victime. Lorsqu'il s'agit de climat empoisonné, la personne est humiliée, disqualifiée, discréditée et isolée. Elle a de la difficulté à s'en remettre parce qu'elle est menacée dans son intégrité.

\section{Harcèlement sexuel}

Le harcèlement sexuel n'est qu'un pas de plus dans les formes de violence pouvant s'exercer. Dans les situations de harcèlement sexuel, «Il ne s'agit pas tant d'obtenir des faveurs de nature sexuelle que de marquer son pouvoir, de considérer la femme comme son objet (sexuel). Une femme harcelée sexuellement est considérée par son agresseur comme étant «à sa disposition» (Hirigoyen 1998: 69). Par conséquent, les femmes devraient accepter. Elles devraient même être flattées ou se sentir heureuses d'avoir été choisies. Lorsqu'il s'agit du harceleur, celui-ci n'envisage pas que la femme puisse dire non. D'ailleurs, si elle le fait, elle subit en retour des humiliations et des agressions.

\section{Agression sexuelle}

Manifestations extrêmes de la violence et du harcèlement sexiste et sexuel, les comportements d'agression sexuelle sont des plus sérieux. Il s'agit d'incidents graves dont les conséquences sur la santé des femmes entraînent des troubles psychologiques, des blessures, des réactions physiques, en plus de mener à des répercussions sociales importantes telles l'isolement et la peur.

Les agressions à caractère sexuel sont des crimes de pouvoir et des actes de violence qui permettent aux hommes d'exercer un contrôle sur la vie des femmes et qui représentent une violation de leurs droits fondamentaux. En d'autres mots, ils perpétuent plus sérieusement, au même titre que les autres formes de harcèlement sexiste et sexuel, les inégalités profondes entre les hommes et les femmes.

Les comportements et les catégories utilisés dans la recherche font partie du continuum de harcèlement sexiste et sexuel. Bien qu'ils ne cernent pas toutes les réalités que recouvrirait une typologie exhaustive, nous croyons qu'ils permettent d'illustrer et de rendre 
compte de la situation qui prévaut dans les institutions d'enseignement postsecondaire comme milieu de travail et d'études.

\section{Méthodologie}

La recherche entreprise par le Comité ad hoc sur la violence en milieu postsecondaire dans le Nord de l'Ontario se divise en deux volets: un sondage par questionnaire et une série de groupes de discussion $^{5}$. Le sondage a été mené à l'aide d'un questionnaire ${ }^{6}$ expédié ou remis à l'ensemble des femmes francophones: étudiantes et apprenantes, membres du personnel administratif et de soutien et membres du corps professoral, qui étudient ou qui travaillent dans les institutions d'éducation postsecondaire francophones ou bilingues du Nord de l'Ontario ${ }^{7}$.

Pour l'ensemble de la population à l'étude, le taux de réponse s'établit à 22,3\% \% . En examinant le taux de réponse selon les différents groupes, on constate que le taux de réponse du personnel de soutien et administratif et du corps professoral (35,8\%) est plus élevé que celui des étudiantes ou apprenantes (19,4\%) (Tableau 1). Il est difficile d'expliquer l'écart important entre eux, mais on peut supposer que depuis la mise en place des politiques institutionnelles en matière de harcèlement, le personnel féminin a une connaissance beaucoup plus grande de ces politiques puisqu'elles ont été distribuées et qu'elles font partie des différentes conventions collectives des travailleuses.

Tableau 1 - Taux de réponse selon les populations féminines francophones et les institutions postsecondaires $^{9}$

\begin{tabular}{lrr} 
& Nombre d'envois & Taux de réponse en \% \\
\hline \hline $\begin{array}{l}\text { Étudiantes et apprenantes } \\
\begin{array}{l}\text { Corps professoral et personnel } \\
\text { de soutien et administratif }\end{array}\end{array}$ & 1979 & $19,4 \%$ \\
\hline Total & 430 & $35,8 \%$ \\
\hline \hline
\end{tabular}


Il s'agit d'un taux de réponse raisonnable compte tenu qu'il s'agissait d'un premier sondage auto-administré par la poste, au contenu relativement long; que l'équipe de recherche n'a pas procédé à une relance systématique auprès des populations à l'étude et, qu'il s'agit d'un phénomène à portée restreinte et d'un sujet encore aujourd'hui largement tabou. En effet, plusieurs études montrent que dans une enquête sur un phénomène comme le harcèlement sexiste et sexuel, seules les personnes touchées ou conscientes de son importance ont tendance à répondre, rendant celui-ci comparable à un échantillonnage de volontaires.

\section{Le portrait de la situation}

\section{Ampleur du harcèlement}

Dans cette recherche, nous avons mesuré l'ampleur du harcèlement à partir des 20 types de comportements de harcèlement vécus par les répondantes. Dans l'ensemble des données recueillies, $68,5 \%$ des répondantes ${ }^{10}$ indiquent avoir subi au moins un comportement de harcèlement sexiste et sexuel dans les 12 mois ayant précédé le sondage. Comme il s'agit d'un premier sondage, il y a lieu de croire que les femmes ont saisi cette opportunité pour briser, de façon anonyme et confidentielle, le silence entourant ce phénomène et pour dire ouvertement les événements qui ont marqué leur vie.

\section{Comportements vécus par les répondantes}

Les répondantes devaient identifier si elles avaient vécu un ou plusieurs des 20 comportements présentés dans le questionnaire. Dans l'année précédant le sondage, on remarque que huit des comportements énumérés ont été vécus plus fréquemment que les autres (Tableau 2). Il s'agit de la réception de messages à contenu sexiste $(84,4 \%)$, de l'exposition à du matériel à caractère sexuel ou pornographique $(80,2 \%)$, de propos sexistes qui abaissent les femmes $(78,6 \%)$, de commentaires non désirés sur la vie sexuelle 
«On ne saurait minimiser l'importance d'un tel climat sexiste. D’apparence bénigne, un climat sexiste masque une réalité plus grave, celle d'un rapport d'infériorité $d u$ sexe féminin. Il crée une ambiance où la femme a toujours la mauvaise partie."
(76,3\%), de blagues sexistes ou d'histoires grossières ou dégradantes $(74,1 \%)$, de regards insistants ou déplacés $(69,1 \%)$, d'intimidation $(68,9 \%)$ et du sentiment d'incompétence lié aux études ou au travail parce que quelqu'un porte atteinte à la réputation dans le but d'abaisser ou de faire du tort (68,9\%).

Quatre des huit comportements dont nous venons de discuter font partie de la catégorie de la situation instaurant un climat sexiste dans les institutions. Or, c'est la catégorie qui obtient le plus grand nombre de réponses, soit 52,5\% ${ }^{11}$ (Tableau 2). On ne saurait minimiser l'importance d'un tel climat sexiste. D'apparence bénigne, un climat sexiste masque une réalité plus grave, celle d'un rapport d'infériorité du sexe féminin. Il crée une ambiance où la femme a toujours la mauvaise partie. Au-delà des propos, des blagues ou des regards insistants, les répondantes se sentent abaissées, choquées, gênées, soulignant leur mal-être. Elles ne se reconnaissent pas dans ces comportements et paroles. Ce sont de petites tracasseries quotidiennes qui fatiguent et qui rappellent sans cesse leur statut inférieur.

Dans la catégorie de comportements instaurant un climat sexiste, l'utilisation du courrier électronique pour transmettre des messages à contenu sexiste est la plus répandue, puisque 84,4\% des répondantes disent avoir reçu de tels messages (Tableau 2). Nous pouvons aisément penser que l'exposition à des photos, des images ou des textes à caractère sexuel ou pornographique offensant $(80,2 \%)$ est du même ordre et passe aussi par ce mode de communication, même si nous considérons ces comportements dans la catégorie du harcèlement sexuel ${ }^{12}$. Ces deux types de comportements semblent choses courantes dans les institutions. Ils le sont d'autant plus que le système de messagerie procure une certaine impunité en garantissant l'anonymat de l'envoyeur à qui veut bien s'en donner la peine. Par ailleurs, dans les douze derniers mois, il semble que le climat sexiste des institutions s'appuie fortement sur les propos sexistes qui abaissent les femmes $(78,6 \%)$, les blagues sexistes et dégradantes $(74,1 \%)$ et les regards déshabilleurs $(69,1 \%)$.

Dans la dernière année, les comportements vécus par les étudiantes ou les apprenantes ou les membres du corps professoral 
ou du personnel administratif et de soutien empoisonnent leur climat d'études ou de travail. En effet, ces comportements obtiennent $24,8 \%$ des réponses et se classent au second rang des plus fortes incidences (Tableau 2). D'ailleurs, tous les comportements de cette catégorie affichent des incidences relativement élevées: sentiment d'incompétence que les femmes éprouvent dans leurs études ou au travail parce que quelqu'un porte atteinte à leur réputation dans le but de les abaisser ou de leur faire du tort $(68,9 \%)$, intimidation qui s'exprime par le haussement de la voix ou par des bêtises ou des injures $(62,4 \%)$, climat d'études ou de travail empoisonné par des propos, des attitudes ou des comportements sexistes (58,3\%) et menaces d'exclusion des études ou de l'emploi (51,2\%).

Les comportements de harcèlement sexuel occupent le troisième rang des réponses $(17,3 \%)$ et les fréquences les plus élevées ont trait à l'exposition à du matériel pornographique ou sexuel offensant (80,2 \%), aux commentaires non désirés sur la vie sexuelle des femmes (76,3\%), aux promesses de récompense en vue de recevoir des faveurs sexuelles (54,5\%) et à l'envoi de cadeaux ou de promesses de bonnes notes en vue de l'obtention de faveurs sexuelles (52,6\%) (Tableau 2). Les répondantes sont aussi les victimes d'invitations répétées afin d'obtenir des faveurs sexuelles $(46,7 \%)$.

Finalement, les comportements d'agression sexuelle obtiennent $5,5 \%$ du total des réponses pour la dernière année (Tableau 2). Les incidences de comportements d'agression sexuelle les plus élevées, même s'ils sont plus rares, sont d'être l'objet d'attouchements sexuels contre son gré $(51,6 \%)$ et la tentative d'agression sexuelle $(45,8 \%)$. 
Tableau 2 - Répartition des comportements non désirés dans les douze mois qui ont précédé l'enquête

\begin{tabular}{|c|c|c|c|}
\hline & Comportements & $\begin{array}{l}\mathrm{N} \\
\text { réponses }\end{array}$ & $\begin{array}{l}\% \text { dans la } \\
\text { catégorie }\end{array}$ \\
\hline \multirow[t]{6}{*}{$\begin{array}{l}\text { Climat sexiste } \\
(52,5 \%)\end{array}$} & $\begin{array}{l}\text { Se faire raconter des blagues sexistes ou des } \\
\text { histoires grossières ou dégradantes qui vous gêne } \\
\text { ou vous choque }\end{array}$ & 203 & 74,1 \\
\hline & $\begin{array}{l}\text { Se faire déshabiller des yeux d'une manière qui vous } \\
\text { gêne ou vous choque }\end{array}$ & 134 & 69,1 \\
\hline & $\begin{array}{l}\text { Entendre quelqu'un tenir des propos sexistes qui } \\
\text { abaissent les femmes }\end{array}$ & 242 & 78,6 \\
\hline & Recevoir des appels téléphoniques sexistes qui vous & & \\
\hline & ont gênée ou choquée & 19 & 32,2 \\
\hline & $\begin{array}{l}\text { Recevoir des lettres ou des messages électroniques } \\
\text { à contenu sexiste qui vous ont gênée ou choquée }\end{array}$ & 103 & 84,4 \\
\hline \multirow[t]{4}{*}{$\begin{array}{l}\text { Climat empoisonné } \\
(24,8 \%)\end{array}$} & $\begin{array}{l}\text { Que quelqu'un vous fasse sentir incompétente dans } \\
\text { vos études ou votre travail afin de porter atteinte à } \\
\text { votre réputation ou dans le but de vous abaisser ou de } \\
\text { vous faire du tort }\end{array}$ & 124 & 68,9 \\
\hline & $\begin{array}{l}\text { Que quelqu'un tente de vous intimider en haussant } \\
\text { la voix ou en vous criant des bêtises ou des insultes }\end{array}$ & 123 & 62,4 \\
\hline & $\begin{array}{l}\text { Que quelqu'un vous menace de vous exclure d'un } \\
\text { cours ou d'un programme d'études ou de vous faire } \\
\text { perdre votre emploi }\end{array}$ & 21 & 51,2 \\
\hline & $\begin{array}{l}\text { Subir un climat d'études ou de travail empoisonné } \\
\text { par des propos, des attitudes ou des comportements } \\
\text { sexistes non désirés }\end{array}$ & 63 & 58,3 \\
\hline \multirow[t]{3}{*}{$\begin{array}{l}\text { Harcèlement } \\
\text { sexuel }(17,3 \%)\end{array}$} & $\begin{array}{l}\text { Que quelqu'un vous fasse des commentaires non } \\
\text { désirés sur votre vie sexuelle }\end{array}$ & 90 & 76,3 \\
\hline & $\begin{array}{l}\text { D'être exposée à des photos, des images ou } \\
\text { des textes à caractère sexuel ou pornographique } \\
\text { offensants dans votre milieu d'études ou de travail }\end{array}$ & 89 & 80,2 \\
\hline & $\begin{array}{l}\text { D'être en présence de quelqu'un qui vous montre } \\
\text { ses organes génitaux sans votre consentement }\end{array}$ & 8 & 36,4 \\
\hline
\end{tabular}


Tableau 2 - Répartition des comportements non désirés dans les douze mois qui ont précédé l'enquête

\begin{tabular}{|c|c|c|c|}
\hline & Comportements & $\begin{array}{c}\mathrm{N} \\
\text { réponses }\end{array}$ & $\begin{array}{l}\% \text { dans la } \\
\text { catégorie }\end{array}$ \\
\hline \multirow[t]{4}{*}{$\begin{array}{l}\text { Harcèlement } \\
\text { sexuel }(17,3 \%)\end{array}$} & $\begin{array}{l}\text { Qu'on vous invite, de façon répétée, afin d'obtenir } \\
\text { des faveurs sexuelles, et ce, malgré votre refus }\end{array}$ & 21 & 46,7 \\
\hline & $\begin{array}{l}\text { De recevoir des cadeaux, des compliments ou des } \\
\text { bonnes notes en vue d'obtenir de vous des faveurs } \\
\text { sexuelles }\end{array}$ & 10 & 52,6 \\
\hline & $\begin{array}{l}\text { De recevoir des avances sexuelles non désirées } \\
\text { accompagnées de promesses de récompense }\end{array}$ & 12 & 54,5 \\
\hline & $\begin{array}{l}\text { De recevoir des avances sexuelles non désirées } \\
\text { accompagnées de menaces de punition }\end{array}$ & 1 & 12,5 \\
\hline \multirow[t]{6}{*}{$\begin{array}{l}\text { Agression sexuelle } \\
(5,5 \%)\end{array}$} & $\begin{array}{l}\text { Que quelqu'un vous touche ou vous caresse dans } \\
\text { le but d'avoir des faveurs sexuelles que vous ne } \\
\text { désiriez pas }\end{array}$ & 33 & 51,6 \\
\hline & $\begin{array}{l}\text { Que quelqu'un tente d'avoir une relation sexuelle } \\
\text { que vous ne désiriez pas }\end{array}$ & 33 & 45,8 \\
\hline & $\begin{array}{l}\text { D'avoir une relation sexuelle sans votre } \\
\text { consentement }\end{array}$ & 6 & 30,0 \\
\hline & $\begin{array}{l}\text { D'avoir été agressée sexuellement avec coups } \\
\text { et blessures }\end{array}$ & 1 & 7,7 \\
\hline & Nombre de réponses & 1336 & - \\
\hline & Nombre de cas & 368 & - \\
\hline
\end{tabular}

Le tableau précédent révèle les aspects les plus importants du harcèlement sexuel et sexiste en milieu de travail ou d'études dans les institutions d'éducation postsecondaires du Nord de la province. Il illustre clairement que les comportements instaurant un climat sexiste ou empoisonné sont ceux auxquels on a davantage recours dans ces institutions. Ils sont les formes les plus courantes de harcèlement sexiste et sexuel.

Par ailleurs, dans le questionnaire, nous avons demandé aux répondantes d'identifier si les comportements qu'elles avaient subis 
s'étaient produits à une seule reprise ou à plusieurs reprises. À cette question, les répondantes ont indiqué que les comportements qui sont vécus fréquemment et de façon répétitive (trois fois ou plus) sont aussi les comportements qui, au tableau précédent, ont les plus fortes fréquences. Il s'agit des propos sexistes qui abaissent les femmes $(30,3 \%)$, des blagues sexistes ou des histoires grossières ou dégradantes $(23,1 \%)$, du sentiment d'incompétence lié aux études ou au travail parce que quelqu'un porte atteinte à la réputation dans le but d'abaisser ou de faire du tort $(13,8 \%)$, de l'intimidation (12,8\%), des regards insistants ou déplacés (12,5\%) et, finalement, des messages à contenu sexiste (12,7\%). Encore ici, l'analyse de la répétition de ces comportements ne fait que montrer que ceux-ci instaurent tout particulièrement un climat sexiste et un climat empoisonné d'études ou de travail dans les institutions.

Ces derniers résultats doivent être analysés avec grande prudence. En effet, d'une part, on pourrait banaliser les comportements qui n'auraient été vécus qu'une seule ou deux fois. D'autre part, en ce qui a trait au critère de répétition, Savoie et Larouche (1988) indiquent qu'il importe de nuancer l'idée largement répandue que le harcèlement doit être soumis au critère de répétition. En effet, ces auteurs indiquent que les comportements de forme physique ou contraignante ne doivent pas nécessairement se répéter afin d'être définis comme du harcèlement au travail. Ils devraient l'être dès leur apparition. De plus, ils ajoutent que si les comportements incluent des représailles, ils doivent alors être

"...s'il est parfois difficile d'identifier le harcèlement sexuel lorsqu'il s'agit d'un seul comportement subtil, il est plus aisé à reconnaître lorsqu'il s'agit de répétition ou de comportements évidents ou grossiers." considérés comme du harcèlement sexuel au travail. Arcand et Archambault vont plus loin en indiquant que le «terme harcèlement implique habituellement l'idée de RÉPÉTITION, toutefois, il peut arriver qu'un seul acte qui engendre un malaise psychologique et physique persistant soit susceptible de provoquer la crainte raisonnable d'une détérioration des conditions de travail» (1993: 3). Ainsi, s'il est parfois difficile d'identifier le harcèlement sexuel lorsqu'il s'agit d'un seul comportement subtil, il est plus aisé à reconnaitre lorsqu'il s'agit de répétition ou de comportements évidents ou grossiers. Pour clore sur la notion de répétition, De Bellefeuille spécifie que cette notion n'a «aucune pertinence dans 
les cas où le harcèlement, même s'il n'a eu lieu qu'une seule fois, a entraîné une perte pour la victime. Cette perte, qu'elle soit salariale ou autre, indique clairement qu'il y a eu dérogation aux procédures normales à cause des préjugés imputables à la situation de harcèlement» (1993:25-26). Faut-il préciser que le milieu d'études est également un lieu de travail?

\section{Personne responsable du harcèlement sexiste et sexuel}

"...les hommes sont les principaux responsables de la situation de harcèlement sexiste et sexuel. »
Le questionnaire permettait aux répondantes d'indiquer la personne responsable du ou des comportements de harcèlement sexiste et sexuel.Elles pouvaient choisir parmi les catégories d'individus suivants: étudiants ou apprenants, professeurs (incluant les instructeurs, superviseurs ou assistants d'enseignement), personnel de soutien ou personnel administratif. De plus, à la question «Par qui ?», le cas échéant, les répondantes pouvaient indiquer qu'elles ne connaissaient pas la personne ou encore qu'il s'agissait d'une personne autre que celles que nous venons de mentionner. Finalement, les répondantes pouvaient inscrire plus d'une réponse, le ou les comportements s'étant souvent répétés, elles peuvent fort bien avoir vécu ces comportements de la part de différentes personnes.

Dans l'ensemble des cas, les hommes sont les principaux responsables de la situation de harcèlement sexiste et sexuel. Ils représentent 74,7 \% de l'ensemble des personnes responsables des situations considérées les plus graves par les répondantes. Dans une proportion beaucoup plus faible, les femmes sont identifiées comme responsables dans $20,3 \%$ des cas et, tout particulièrement, dans les comportements instaurant un climat empoisonné.

Le tableau 3 fait état de la répartition des comportements de harcèlement sexiste et sexuel vécus par les répondantes selon la fonction sociale de la personne qui en est responsable.

\section{Les étudiants et les apprenants}

Les comportements des étudiants et des apprenants les plus fréquents sont de nature à créer un climat sexiste: raconter des blagues sexistes et des histoires grossières ou dégradantes (46,9\%), déshabiller des yeux de façon gênante ou choquante (40,3\%), 
tenir des propos sexistes qui abaissent les femmes (44,5\%). Ils sont aussi les principaux initiateurs des comportements de harcèlement sexuel et, plus particulièrement, lorsqu'il s'agit d'émettre des commentaires non désirés sur la vie sexuelle (51,5\%). Finalement, les étudiants et les apprenants sont aussi les principaux responsables de comportements d'agression sexuelle: les attouchements sexuels contre son gré (46\%) et les tentatives d'agression sexuelle $(39,7 \%)$ (Tableau 3$)$.

\section{Le corps professoral}

Le corps professoral est identifié comme étant le principal responsable du climat empoisonné dans les institutions de l'étude. Dans cette catégorie, il est le principal instigateur des quatre comportements de cette catégorie. Les incidences sont les suivantes: sentiment d'incompétence que les femmes éprouvent dans leurs études ou au travail parce que quelqu'un porte atteinte à leur réputation dans le but de les abaisser ou de leur faire du tort $(44,5 \%)$, climat empoisonné par des propos, des attitudes ou des comportements sexistes (42,2\%), menaces d'exclusion d'un cours, d'un programme ou d'un emploi (40 \%) et, finalement, intimidation qui s'exprime par le haussement de la voix ou par des bêtises ou des injures (31,2\%). Pour les comportements de harcèlement sexuel, le corps professoral se situe au second rang pour l'utilisation des avances sexuelles non désirées et accompagnées de menaces de punition (25\%) (Tableau 3).

\section{Le personnel administratif et de soutien}

Le personnel administratif et de soutien n'est pas le principal instigateur des comportements de harcèlement sexiste et sexuel dans les institutions. Par contre, il se situe au second rang dans la mise en place d'un climat empoisonné lorsqu'il s'agit d'utiliser les comportements menaçants d'exclusion $(37,8 \%)$ et les comportements liés au développement d'un sentiment d'incompétence (25,4\%) (Tableau 3).

\section{Et les autres responsables...}

Les comportements pour lesquels les répondantes connaissent les 
auteurs sont importants et affectent leur vie au travail ou aux études. Plus particulièrement, il s'agit de comportements de harcèlement sexuel et d'agression sexuelle qui sont perpétrés, dans une large proportion, par d'autres personnes connues des répondantes (Tableau 3). Si les personnes autres que celles qui forment le personnel ou le corps étudiant ou apprenant des institutions sont identifiées comme étant responsables des comportements graves de harcèlement sexiste et sexuel, cela ne procure pas une licence pour ne rien faire. Les universités et les collèges ne sont pas des milieux clos. La vie étudiante ou apprenante, comme la vie professionnelle, offrent de nombreuses occasions de rencontrer des personnes qui n'ont pas de rapports directs avec l'institution. Par conséquent, même si nous limitons l'article à la problématique du harcèlement dans le cadre du travail ou des études, il ne faut pas non plus se dégager de la responsabilité collective qui nous incombe de venir en aide et d'appuyer les femmes ayant un semblable vécu.

Tableau 3 - Répartition des comportements de harcèlement sexiste et sexuel selon la personne responsable du comportement (en pourcentage)

\begin{tabular}{|c|c|c|c|c|c|}
\hline Comportements de harcèlement sexiste et sexuel & $\begin{array}{l}\text { Étudiants - } \\
\text { apprenants }\end{array}$ & $\begin{array}{l}\text { Membres } \\
\text { du corps } \\
\text { professoral }\end{array}$ & $\begin{array}{l}\text { Membres } \\
\text { du personnel } \\
\text { de soutien - } \\
\text { administratif }\end{array}$ & $\begin{array}{l}\text { Ne sais } \\
\text { pas }\end{array}$ & $\begin{array}{c}\text { Autre } \\
\text { personne }\end{array}$ \\
\hline $\begin{array}{l}\text { Se faire raconter des blagues sexistes ou des } \\
\text { histoires grossières ou dégradantes qui vous } \\
\text { gêne ou vous choque }\end{array}$ & 46,9 & 23,4 & 16,3 & 3,1 & 10,2 \\
\hline $\begin{array}{l}\text { Se faire déshabiller des yeux d'une manière } \\
\text { qui vous gêne ou vous choque }\end{array}$ & 40,3 & 25,9 & 9,0 & 11,1 & 13,6 \\
\hline $\begin{array}{l}\text { Entendre quelqu'un tenir des propos sexistes } \\
\text { qui abaissent les femmes }\end{array}$ & 44,5 & 23,3 & 13,6 & 7,4 & 11,3 \\
\hline $\begin{array}{l}\text { Recevoir des appels téléphoniques sexistes } \\
\text { qui vous ont gênée ou choquée }\end{array}$ & 10,0 & 6,7 & 5,0 & 61,7 & 16,7 \\
\hline $\begin{array}{l}\text { Recevoir des lettres ou des messages électro- } \\
\text { niques à contenu sexiste qui vous ont gênée } \\
\text { ou choquée }\end{array}$ & 32,5 & 11,0 & 16,2 & 20,1 & 20,1 \\
\hline
\end{tabular}


Tableau 3 - Répartition des comportements de harcèlement sexiste et sexuel selon la personne responsable du comportement (suite)

\begin{tabular}{|c|c|c|c|c|c|}
\hline Comportements de harcèlement sexiste et sexuel & $\begin{array}{l}\text { Étudiants - } \\
\text { apprenants }\end{array}$ & $\begin{array}{l}\text { Membres } \\
\text { du corps } \\
\text { professoral }\end{array}$ & $\begin{array}{l}\text { Membres } \\
\text { du personnel } \\
\text { de soutien - } \\
\text { administratif }\end{array}$ & $\begin{array}{l}\text { Ne sais } \\
\text { pas }\end{array}$ & $\begin{array}{c}\text { Autre } \\
\text { personne }\end{array}$ \\
\hline $\begin{array}{l}\text { Que quelqu'un vous fasse sentir incompétente } \\
\text { dans vos études ou votre travail afin de porter } \\
\text { atteinte à votre réputation ou dans le but de } \\
\text { vous abaisser ou de vous faire du tort }\end{array}$ & 21,2 & 44,5 & 25,4 & 0,4 & 8,5 \\
\hline $\begin{array}{l}\text { Que quelqu'un tente de vous intimider en } \\
\text { haussant la voix ou en vous criant des bêtises } \\
\text { ou des insultes }\end{array}$ & 28,6 & 31,2 & 19,2 & 3,4 & 17,5 \\
\hline $\begin{array}{l}\text { Que quelqu'un vous menace de vous exclure } \\
\text { d'un cours ou d'un programme d'études ou de } \\
\text { vous faire perdre votre emploi }\end{array}$ & 2,2 & 40,0 & 37,8 & 2,2 & 17,8 \\
\hline $\begin{array}{l}\text { Subir un climat d'études ou de travail empoi- } \\
\text { sonné par des propos, des attitudes ou des } \\
\text { comportements sexistes non désirés }\end{array}$ & 26,8 & 42,2 & 19,0 & 0,7 & 11,3 \\
\hline $\begin{array}{l}\text { Que quelqu'un vous fasse des commentaires } \\
\text { non désirés sur votre vie sexuelle }\end{array}$ & 51,5 & 8,9 & 13,4 & 6,7 & 19,4 \\
\hline $\begin{array}{l}\text { D'être exposée à des photos, des images ou } \\
\text { des textes à caractère sexuel ou pornogra- } \\
\text { phique offensants dans votre milieu d'études } \\
\text { ou de travail }\end{array}$ & 33,1 & 13,9 & 22,3 & 7,7 & 23,1 \\
\hline $\begin{array}{l}\text { D'être en présence de quelqu'un qui vous } \\
\text { montre ses organes génitaux sans votre } \\
\text { consentement }\end{array}$ & 35,0 & - & - & 5,0 & 60,0 \\
\hline $\begin{array}{l}\text { Qu'on vous invite, de façon répétée, afin } \\
\text { d'obtenir des faveurs sexuelles, et ce, malgré } \\
\text { votre refus }\end{array}$ & 44,7 & 14,9 & 6,4 & - & 34,0 \\
\hline $\begin{array}{l}\text { De recevoir des cadeaux, des compliments ou } \\
\text { des bonnes notes en vue d'obtenir de vous } \\
\text { des faveurs sexuelles }\end{array}$ & 40,0 & 10,0 & 5,0 & - & 45,0 \\
\hline $\begin{array}{l}\text { De recevoir des avances sexuelles non désirées } \\
\text { accompagnées de promesses de récompense }\end{array}$ & 33,3 & 12,5 & 8,4 & 8,3 & 37,5 \\
\hline
\end{tabular}


Tableau 3 - Répartition des comportements de harcèlement sexiste et sexuel selon la personne responsable du comportement (suite)

\begin{tabular}{|c|c|c|c|c|c|}
\hline Comportements de harcèlement sexiste et sexuel & $\begin{array}{l}\text { Étudiants - } \\
\text { apprenants }\end{array}$ & $\begin{array}{l}\text { Membres } \\
\text { du corps } \\
\text { professoral }\end{array}$ & $\begin{array}{c}\text { Membres } \\
\text { du personnel } \\
\text { de soutien - } \\
\text { administratif }\end{array}$ & $\begin{array}{l}\text { Ne sais } \\
\text { pas }\end{array}$ & $\begin{array}{c}\text { Autre } \\
\text { personne }\end{array}$ \\
\hline $\begin{array}{l}\text { De recevoir des avances sexuelles non désirées } \\
\text { accompagnées de menaces de punition }\end{array}$ & - & 25,0 & 25,0 & - & 50,0 \\
\hline $\begin{array}{l}\text { Que quelqu'un vous touche ou vous caresse } \\
\text { dans le but d'avoir des faveurs sexuelles que } \\
\text { vous ne désiriez pas }\end{array}$ & 46,0 & 9,5 & 6,4 & 4,8 & 38,1 \\
\hline $\begin{array}{l}\text { Que quelqu'un tente d'avoir une relation } \\
\text { sexuelle que vous ne désiriez pas }\end{array}$ & 39,7 & 10,9 & 4,1 & 5,4 & 39,7 \\
\hline $\begin{array}{l}\text { D'avoir une relation sexuelle sans votre } \\
\text { consentement }\end{array}$ & 26,3 & - & - & - & 73,7 \\
\hline $\begin{array}{l}\text { D'avoir été agressée sexuellement avec coups } \\
\text { et blessures }\end{array}$ & 28,6 & - & - & 7,1 & 64,3 \\
\hline
\end{tabular}

Les pourcentages sont basés sur le nombre de réponses.

Comme l'enquête portait principalement sur le harcèlement sexiste et sexuel dans les institutions d'enseignement postsecondaire, nous avons voulu connaître comment se tissent les relations entre les trois groupes occupant des fonctions différentes au sein de ces institutions, soit les étudiants et les étudiantes ou les apprenants et les apprenantes, le corps professoral et le personnel administratif et de soutien. Dans l'ensemble des données, dans l'année qui a précédé le sondage ${ }^{13}$, il se dessine plusieurs tendances relationnelles de harcèlement.

\section{Un milieu ambiant empreint de sexisme pour les étudiantes et les apprenantes}

Un regard sur les relations entre les étudiantes et les apprenantes et leurs homologues masculins montre que ces derniers occupent le premier rang lorsqu'il s'agit d'instaurer un climat sexiste en 
"Dans le corps

étudiant, le

harcèlement est

horizontal, c'est-à-dire

que les étudiants et les

apprenants instaurent

un climat sexiste ou

empoisonné à l'égard

des étudiantes et des

apprenantes. " milieu d'études universitaires ou collégiales (Tableau 4). Non seulement, les étudiants et les apprenants instaurent-ils un climat sexiste mais ils sont aussi la source de nombreux comportements instaurant un climat empoisonné en milieu d'études (Tableau 5). Par ailleurs, même si les comportements de harcèlement sexuel ou d'agression sexuelle sont dénoncés dans une moins grande fréquence que les comportements sexistes ou empoisonnés, il n'en reste pas moins que les étudiantes et les apprenantes indiquent qu'ils sont principalement le fait de leurs collègues masculins (Tableaux 6 et 7).

Dans le corps étudiant, le harcèlement est horizontal, c'est-àdire que les étudiants et les apprenants instaurent un climat sexiste ou empoisonné à l'égard des étudiantes et des apprenantes. Il y a très peu de conduites de ce genre qui sont dirigées vers le corps professoral ou vers le personnel de soutien et administratif.

Plus généralement, ces conduites ne semblent reproduire que le sexisme qu'ils ont appris dans leur enfance ou leur adolescence. Et ce sexisme illustre leur vision de la condition féminine.

Pour les étudiantes et les apprenantes soumises à ce climat sexiste, les conséquences de tels comportements sont principalement d'ordre psychologique (colère, stress, nervosité, angoisse ou anxiété, perte de confiance en soi). Celles-ci sont suivies de conséquences sur les études (difficultés de concentration, perte de motivation) puis, des conséquences sur la santé physique (fatigue, maux de tête, insomnie). Les étudiantes et les apprenantes mentionnent aussi des conséquences sur leur vie sociale et personnelle. Elles perdent confiance dans les autres, évitent dorénavant certains lieux, $\mathrm{s}^{\prime}$ isolent ${ }^{14}$, etc. Ce climat sexiste nuit à l'égalité et ne constitue guère un milieu d'apprentissage propice à l'épanouissement personnel.

\section{Un climat empoisonné pour le personnel de soutien ou le personnel administratif}

Les données du sondage révèlent qu'en milieu de travail, les employées du personnel administratif et de soutien identifient d'abord leurs collègues comme étant les responsables de 
"Les membres faisant partie du personnel de soutien ou administratif indiquent que les principaux comportements dont elles sont victimes de la part de leurs pairs sont de l'ordre du climat empoisonné de travail." comportements de harcèlement.Toutefois, dans le cas du personnel de soutien et administratif, il peut s'agir de harcèlement horizontal (entre collègues) ou de harcèlement vertical, d'une personne de niveau hiérarchique supérieur envers une personne subordonnée. Mais, en regroupant les membres du personnel au sein des divers établissements, nous nous sommes privées de pouvoir identifier la relation d'autorité qui pouvait être en cause. Ce serait une étude à poursuivre.

Les membres faisant partie du personnel de soutien ou administratif indiquent que les principaux comportements dont elles sont victimes de la part de leurs pairs sont de l'ordre du climat empoisonné de travail (Tableau 5). En second lieu, elles montrent que ceux-ci sont suivis par les comportements instaurant un climat sexiste dans leur milieu de travail (Tableau 4). Par contre, elles disent aussi être occasionnellement victimes de comportements sexistes ou empoisonnés de la part du corps professoral.

Dans les institutions à l'étude, nous ne connaissons pas les raisons associées au harcèlement en milieu de travail. Toutefois, d'un côté, Dejours (1988), Soares (2002) et Debout et Larose (2003) pointent tous vers la déshumanisation du travail, vers les nouveaux rapports de travail qui sont caractérisés par l'affaiblissement des collectifs de travail, la déstructuration des solidarités causée par les nouvelles formes d'organisation du travail axées sur l'individualisation, et l'isolement des individus dans le temps et l'espace. Ce serait cette transformation de l'organisation et des conditions de travail qui serait la cause de la recrudescence du harcèlement au travail. De l'autre côté, Gauvin (1991) et le Conseil du statut de la femme du Québec (1995) pointent davantage vers le sexisme comme explication au harcèlement en milieu d'éducation postsecondaire. Souvent, l'une ou l'autre se conjuguent dans l'explication. Toutefois, quelle que soit celle que l'on choisisse ou que l'on promeut, les conséquences individuelles sur le personnel de soutien et administratif, comme les conséquences sur l'organisation, sont néfastes: l'absentéisme, le manque d'initiative, la rétention d'information, la diffamation, etc. 


\section{Un climat empoissonné par le corps professoral}

«...les étudiantes et les apprenantes indiquent que le corps professoral occupe le premier rang lorsqu'il s'agit d'utiliser des comportements instaurant un climat empoisonné envers eux. "
Dans le sondage, les étudiantes et les apprenantes indiquent que le corps professoral occupe le premier rang lorsqu'il s'agit d'utiliser des comportements instaurant un climat empoisonné envers eux (Tableau 5). Le corps professoral étant en position hiérarchique, le harcèlement devient alors un espace où l'autorité du professeur menace ou compromet l'apprentissage de l'étudiante ou de l'apprenante. En fait, les principaux comportements dénoncés contribuant à installer un climat empoisonné cherchent à diminuer, à souligner l'incompétence de l'apprenante ou de l'étudiante, à attaquer sa réputation. Dans un second temps, les comportements dénoncés sont de l'ordre de l'intimidation en haussant la voix ou en criant des bêtises.

Dans la mesure où, dans le cas des étudiantes et des apprenantes, les comportements instaurant un climat empoisonné se produisent sur les lieux des institutions, nous pouvons présumer que la mise en place d'un climat hostile, menaçant ou offensant à leur égard a comme effet de les déprécier, de les dénigrer et de les exclure de l'institution. La présence de tels comportements vise à les décourager d'affirmer leur droit à la non discrimination et démontre que beaucoup de travail reste à faire. L'imposition d'un tel climat doit être sérieusement prise en considération compte tenu de la présence croissante de la population étudiante féminine dans les établissements d'enseignements postsecondaires. 
Tableau 4-Comportements instaurant un climat sexiste, selon le statut des protagonistes (12 derniers mois avant le sondage)

\begin{tabular}{|c|c|c|c|c|c|}
\hline \multirow{2}{*}{\multicolumn{2}{|c|}{ CLIMAT SEXISTE }} & \multicolumn{4}{|c|}{ STATUT DE LA VICTIME } \\
\hline & & Étudiantes & Corps & & Total \\
\hline \multirow{4}{*}{$\begin{array}{l}\text { Statut de la } \\
\text { personne } \\
\text { responsable du } \\
\text { comportement }\end{array}$} & Étudiants ou apprenants & $\begin{array}{r}422 \\
87,9 \\
73,0 \\
47,9\end{array}$ & $\begin{array}{r}22 \\
4,6 \\
31,0 \\
2,5\end{array}$ & $\begin{array}{r}36 \\
7,5 \\
15,5 \\
4,1\end{array}$ & $\begin{array}{r}480 \\
54,5\end{array}$ \\
\hline & Corps professoral & $\begin{array}{r}133 \\
55,0 \\
23,0 \\
15,1\end{array}$ & $\begin{array}{r}28 \\
11,6 \\
39,4 \\
3,2\end{array}$ & $\begin{array}{r}81 \\
33,5 \\
34,9 \\
9,2\end{array}$ & $\begin{array}{r}242 \\
27,5\end{array}$ \\
\hline & $\begin{array}{l}\text { Personnel de soutien } \\
\text { et administratif }\end{array}$ & $\begin{array}{r}23 \\
14,5 \\
4,0 \\
2,6\end{array}$ & $\begin{array}{r}21 \\
13,2 \\
29,6 \\
2,4\end{array}$ & $\begin{array}{r}115 \\
72,3 \\
49,6 \\
13,1\end{array}$ & $\begin{array}{r}159 \\
18,0\end{array}$ \\
\hline & Total & $\begin{array}{r}578 \\
65,6\end{array}$ & $\begin{array}{r}71 \\
8,1\end{array}$ & $\begin{array}{r}232 \\
26,3\end{array}$ & $\begin{array}{r}881 \\
100,0\end{array}$ \\
\hline
\end{tabular}

Les pourcentages sont basés sur le nombre des réponses - nombre de cas $=302$ 
Tableau 5 - Comportements instaurant un climat empoisonné selon le statut des protagonistes (12 derniers mois avant le sondage)

\begin{tabular}{|c|c|c|c|c|c|}
\hline \multirow{2}{*}{\multicolumn{2}{|c|}{ CLIMAT EMPOISONNÉ }} & \multicolumn{4}{|c|}{ STATUT DE LA VICTIME } \\
\hline & & Étudiantes & Corps & Personnel de & Total \\
\hline \multirow{4}{*}{$\begin{array}{l}\text { Statut de la } \\
\text { personne } \\
\text { responsable du } \\
\text { comportement }\end{array}$} & Étudiants ou apprenants & $\begin{array}{r}123 \\
84,8 \\
41,0 \\
24,3\end{array}$ & $\begin{array}{r}7 \\
4,8 \\
17,5 \\
1,4\end{array}$ & $\begin{array}{r}15 \\
10,3 \\
9,0 \\
3,0\end{array}$ & $\begin{array}{r}145 \\
28,6\end{array}$ \\
\hline & Corps professoral & $\begin{array}{r}145 \\
64,2 \\
48,3 \\
28,6\end{array}$ & $\begin{array}{r}23 \\
10,2 \\
57,5 \\
4,5\end{array}$ & $\begin{array}{r}58 \\
25,7 \\
34,7 \\
11,4\end{array}$ & $\begin{array}{r}226 \\
44,6\end{array}$ \\
\hline & $\begin{array}{l}\text { Personnel de soutien } \\
\text { et administratif }\end{array}$ & $\begin{array}{r}32 \\
23,5 \\
10,7 \\
6,3 \\
\end{array}$ & $\begin{array}{r}10 \\
7,4 \\
25,0 \\
2,0\end{array}$ & $\begin{array}{r}94 \\
69,1 \\
56,3 \\
18,5\end{array}$ & $\begin{array}{r}136 \\
26,8\end{array}$ \\
\hline & Total & $\begin{array}{r}300 \\
59,2\end{array}$ & $\begin{array}{r}40 \\
7,9\end{array}$ & $\begin{array}{r}167 \\
32,9\end{array}$ & $\begin{array}{r}507 \\
100,0\end{array}$ \\
\hline
\end{tabular}

Les pourcentages sont basés sur le nombre des réponses - nombre de cas $=225$

Les tableaux 6 et 7 qui suivent méritent une mise en garde. En effet, les nombres de cas sont beaucoup moins importants lorsqu'il s'agit de harcèlement sexuel ou d'agression sexuelle que lorsqu'il s'agit de climat sexiste ou de climat empoisonné. En ce sens, il devient difficile de généraliser les résultats. Malgré la faiblesse des nombres, ils peuvent être révélateurs qu'il y ait anguille sous roche. En raison de la gravité des comportements constatés, on doit certes leur accorder toute l'importance qu'ils méritent dans les institutions d'éducation postsecondaire.

Pour les comportements de harcèlement sexuel, nous avons déjà souligné qu'ils étaient davantage le fait des étudiants ou des apprenants (58,6\% des réponses). Par contre, les relations de travail au sein du personnel de soutien et administratif sont entachées 
par de tels comportements de la part du personnel de soutien et administratif envers leurs pairs (13,0\%). Plus rarement, ils sont aussi le fait du corps professoral à l'égard des étudiantes ou des apprenantes $(6,7 \%)$ ou à l'égard du personnel de soutien $(5,9 \%)$ pour un total de 12,6\% des réponses. Par ailleurs, si les comportements d'agression sexuelle sont principalement exercés par les étudiants et les apprenants (70,9\% des réponses), il importe de souligner qu'occasionnellement, ils sont le fait du corps professoral ou du personnel administratif ou de soutien à l'égard du personnel de soutien ( 6,3 et $5,1 \%$ des réponses respectivement).

Tableau 6-Comportements de harcèlement sexuel selon le statut des protagonistes (12 derniers mois avant le sondage)

\begin{tabular}{|c|c|c|c|c|c|}
\hline \multirow{2}{*}{\multicolumn{2}{|c|}{ HARCÈLEMENT SEXUEL }} & \multicolumn{4}{|c|}{ STATUT DE LA VICTIME } \\
\hline & & \multirow{2}{*}{$\begin{array}{r}\begin{array}{r}\text { Étudiantes } \\
\text { apprenantes }\end{array} \\
140 \\
90,3 \\
83,8 \\
58,6\end{array}$} & \multirow{2}{*}{$\begin{array}{r}\begin{array}{r}\text { Corps } \\
\text { professoral }\end{array} \\
4 \\
2,6 \\
25,0 \\
1,7\end{array}$} & \multirow{2}{*}{ 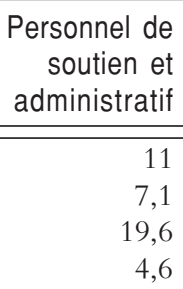 } & \multirow{2}{*}{$\begin{array}{r}\text { Total } \\
155 \\
64,9\end{array}$} \\
\hline \multirow{4}{*}{$\begin{array}{l}\text { Statut de la } \\
\text { personne } \\
\text { responsable du } \\
\text { comportement }\end{array}$} & Étudiants ou apprenants & & & & \\
\hline & Corps professoral & $\begin{array}{r}16 \\
42,1 \\
9,6 \\
6,7\end{array}$ & $\begin{array}{r}8 \\
21,1 \\
50,0 \\
3,3\end{array}$ & $\begin{array}{r}14 \\
36,8 \\
25,0 \\
5,9\end{array}$ & $\begin{array}{r}38 \\
15,9\end{array}$ \\
\hline & $\begin{array}{l}\text { Personnel de soutien } \\
\text { et administratif }\end{array}$ & $\begin{array}{r}11 \\
23,9 \\
6,6 \\
4,6\end{array}$ & $\begin{array}{r}4 \\
8,7 \\
25,0 \\
1,7\end{array}$ & $\begin{array}{r}31 \\
67,4 \\
55,4 \\
13,0\end{array}$ & $\begin{array}{r}46 \\
19,2\end{array}$ \\
\hline & Total & $\begin{array}{r}167 \\
69,9\end{array}$ & $\begin{array}{r}16 \\
6,7\end{array}$ & $\begin{array}{r}56 \\
23,4\end{array}$ & $\begin{array}{r}239 \\
100,0\end{array}$ \\
\hline
\end{tabular}

Les pourcentages sont basés sur le nombre des réponses - nombre de cas $=153$ cas 
Tableau 7 - Comportement d'agression sexuelle selon le statut des protagonistes (12 derniers mois avant le sondage)

\begin{tabular}{|c|c|c|c|c|c|}
\hline \multirow{2}{*}{\multicolumn{2}{|c|}{ AGRESSION SEXUELLE }} & \multicolumn{4}{|c|}{ STATUT DE LA VICTIME } \\
\hline & & \multirow{2}{*}{$\begin{array}{r}\begin{array}{r}\text { Étudiantes } \\
\text { apprenantes }\end{array} \\
56 \\
90,3 \\
94,9 \\
70,9\end{array}$} & \multirow{2}{*}{$\begin{array}{r}\text { Corps } \\
\text { professoral } \\
6 \\
9,7 \\
54,5 \\
7,6\end{array}$} & \multirow{2}{*}{ 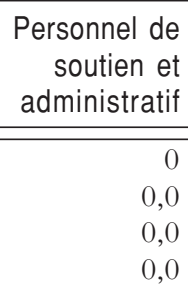 } & \multirow{2}{*}{$\begin{array}{r}\text { Total } \\
62 \\
78,5\end{array}$} \\
\hline \multirow{4}{*}{$\begin{array}{l}\text { Statut de la } \\
\text { personne } \\
\text { responsable du } \\
\text { comportement }\end{array}$} & Étudiants ou apprenants & & & & \\
\hline & Corps professoral & $\begin{array}{r}2 \\
18,2 \\
3,4 \\
2,5\end{array}$ & $\begin{array}{r}4 \\
36,4 \\
36,4 \\
5,1\end{array}$ & $\begin{array}{r}5 \\
45,6 \\
55,6 \\
6,3\end{array}$ & $\begin{array}{r}11 \\
13,9\end{array}$ \\
\hline & $\begin{array}{l}\text { Personnel de soutien } \\
\text { et administratif }\end{array}$ & $\begin{array}{r}1 \\
16,7 \\
1,7 \\
1,3\end{array}$ & $\begin{array}{r}1 \\
16,7 \\
1,7 \\
1,3\end{array}$ & $\begin{array}{r}4 \\
66,7 \\
44,4 \\
5,1\end{array}$ & $\begin{array}{r}6 \\
7,6\end{array}$ \\
\hline & Total & $\begin{array}{r}59 \\
74,7\end{array}$ & $\begin{array}{r}11 \\
13,9\end{array}$ & $\begin{array}{r}9 \\
11,4\end{array}$ & $\begin{array}{r}79 \\
100,0\end{array}$ \\
\hline
\end{tabular}

Les pourcentages sont basés sur le nombre des réponses - nombre de cas $=54$

\section{Un malaise dans les institutions d'éducation}

Le harcèlement sexiste et sexuel, révélé ici, n'est pas différent d'autres institutions d'éducation ou d'autres milieux de travail. Les données sur les comportements de harcèlement sexiste et sexuel, tout comme celles portant sur les relations interpersonnelles, traduisent un malaise au sein d'institutions où les gens se côtoient quotidiennement et où on se ferme les yeux pour ne pas le voir.

Certes, une mise en garde s'impose. Comme l'indiquent Leymann (1996), Hirigoyen (2001) ou Debout et Larose (2003), 
"Il importe de distinguer nettement ce qui est ou n'est pas du harcèlement, de bien cerner ce qui tient $d u$ stress au travail ou dans les études, des désaccords, des conflits, des contraintes professionnelles, des pressions de certains dirigeants, bref, de tous les glissements possibles..."

"Et si les plaintes se font rares, c'est que la peur s'installe permettant au harcèlement sexiste et sexuel de perdurer." il faut être vigilant, pouvoir démêler le vrai du faux. Il importe de distinguer nettement ce qui est ou n'est pas du harcèlement, de bien cerner ce qui tient du stress au travail ou dans les études, des désaccords, des conflits, des contraintes professionnelles, des pressions de certains dirigeants, bref, de tous les glissements possibles qui pourraient amener une travailleuse ou une étudiante à qualifier de harcèlement, d'autres souffrances au travail ou aux études. En ce sens, la présente étude gagnerait à être poursuivie afin de bien saisir ces nuances. Mais, à l'inverse, il ne faudrait pas non plus que le manque de vigilance nuise aux réelles victimes de harcèlement sexiste et sexuel.

Au-delà de cette mise en garde, le malaise dont nous parlons existe bien. Phénomène inavouable, le harcèlement sexiste et sexuel trouve sa source dans le refus de respecter l'autre, de l'accueillir dans ses différences: celles du sexe principalement, mais aussi du caractère, des comportements, de la race, de la culture, de la classe sociale... Questions de jalousie, d'envie, de rivalité, de rejet, de mépris, d'indifférence, toutes ces facettes jouent un rôle dans un monde du travail où elles deviennent possibles en raison de la disparition des collectifs de travail et de la déshumanisation du travail (Debout et Larose 2003) ou par le pouvoir de la discrimination et du sexisme ambiants. Dans le milieu postsecondaire où les femmes font de plus en plus leur place dans la structure du travail ou dans celle de l'éducation, elles sont marginalisées, renvoyées aux stéréotypes par le biais du harcèlement sexiste et sexuel exercé par des hommes et, occasionnellement, reconduit par des femmes.

Et si les plaintes se font rares, c'est que la peur s'installe permettant au harcèlement sexiste et sexuel de perdurer. Pour la victime et pour le harceleur, la peur est présente, mais elle est bien différente. Pour les victimes, le fait d'en parler à quelqu'un ne suffit pas à les inciter à porter plainte ou à chercher de l'aide. Il faut que la situation devienne intenable avant de la dénoncer et même là, les femmes vont chercher à la régler par elles-mêmes. Dans le sondage mené, les répondantes disent qu'elles discutent de leur situation de harcèlement avec une ou plusieurs personnes (la famille, les amies et amis, les collègues), mais n'osent pas 
"...la peur que l'on a pour soi et en soi sert de ressort pour attaquer l'autre. Et avec la peur vient aussi la honte d'avoir peur, la lâcheté et les comportements infantiles. " officiellement dénoncer leur harceleur. Lorsqu'on regroupe les différentes raisons pour ne pas porter plainte, les répondantes disent qu'elles doivent soit prendre elles-mêmes le problème en main ${ }^{15}$, soit qu'elles ont peur des réactions de l'environnement: elles craignent les réactions, les représailles de l'entourage; elles ont peur d'être accusées de provocation; elles pensent que leur situation ne sera pas prise au sérieux; elles craignent de compromettre leurs études ou leur emploi. D'autres répondantes justifient autrement le fait de ne pas porter plaine: elles invoqueront une série de raisons liées aux difficultés qu'elles entrevoient face au dépôt d'une plainte (manque de confiance envers les personnes en place, démarches compliquées, ne pas savoir à qui s'adresser, se faire déconseiller de porter plainte $)^{16}$.

Du point de vue du harceleur, Hirigoyen (2001) souligne que la peur est le «moteur essentiel du harcèlement» car c'est par peur qu'on devient violent: peur de ne pas être à la hauteur, peur de déplaire, peur de ne pas être apprécié, peur du changement, peur d'une sanction ou peur d'une faute professionnelle. Bref, la peur que l'on a pour soi et en soi sert de ressort pour attaquer l'autre. Et avec la peur vient aussi la honte d'avoir peur, la lâcheté et les comportements infantiles.

\section{Conclusion}

L'analyse des données sur le harcèlement sexiste et sexuel dans les institutions d'éducation postsecondaire montre qu'au-delà des données factuelles, les différents comportements se réferent à un climat de travail sexiste et empoisonné. Tout se joue comme si ce climat de travail et d'études était «institué», qu'il a infiltré toutes les facettes des institutions. Ce constat va à l'encontre de ce qu'on pourrait penser des institutions d'éducation postsecondaire, c'està-dire qu'elles ont été mises en place pour favoriser l'épanouissement de l'expérience individuelle et collective et non, pour la brimer. Plus particulièrement, pour les étudiantes et les apprenantes, il importe non pas de dénoncer leurs incompétences 
mais de les soutenir dans leur démarche éducative. On s'attendrait davantage à voir se développer un soutien pour leur faciliter le passage vers une vie professionnelle pour laquelle elles auront acquis les meilleurs atouts possibles.

Un climat sexiste règne aussi dans nos institutions postsecondaires où nous nous flattons d'être à l'avant-garde des courants de pensée qui animent nos sociétés. En effet, quelle est la vérité-message qui se cache derrière ces plaisanteries et cet «humour» choquant, abaissant aux dires des répondantes, si ce n'est celle de l'infériorité de statut du sexe féminin?

Il est important d'ouvrir les yeux, de confronter du regard et de dénoncer cette situation. Les hommes et les femmes peuvent contribuer à créer un climat exempt de harcèlement. Le travail ou les études sont des lieux de réalisation de soi tant individuelle que collective. Dans ce contexte, qu'on promeuve l'individualisme, source de création, il en va de soi. Mais la promotion de l'individualisme ne doit pas se faire au profit de l'abaissement de l'«Autre» par des pratiques de harcèlement, sinon le collectif en sort perdant en épuisant ses réserves de solidarité. Il est aussi du ressort des institutions de promouvoir un climat exempt de ce type de comportements.

\section{Bibliographie}

ARCAND, D. et D. ARCHAMBAULT (1993). Le harcèlement sexuel au travail doit cesser afin que les femmes puissent travailler en paix et ainsi donner la pleine mesure de leur compétence, Trois-Rivières, Calacs.

CANTIN, S et C. PROULX (1995). À l'Université comme ailleurs. Rapport du sondage sur le harcèlement sexuel mené à l'Université de Montréal, Montréal, Université de Montréal, Bureau d'intervention en matière de harcèlement sexuel.

CONSEIL DU STATUT DE LA FEMME (1995). La violence faite aux femmes à travers les agressions sexuelles, Québec, Gouvernement du Québec, Conseil du statut de la femme.

COMMISSION CANADIENNE DES DROITS DE LA PERSONNE (1998). Rapport final remis à la Commission canadienne des droits de la personne, Ottawa, Commission canadienne des droits de la personne.

COMMISSION DES DROITS DE LA PERSONNE DU NOUVEAU-BRUNSWICK. (Page consultée le 12 novembre 2001). Harcèlement sexuel, [En ligne], Adresse URL: http:// www.ajefnb.nb.ca/guidejur/sexuel.html 
COMMISSION ONTARIENNE DES DROITS DE LA PERSONNE. (Page consultée le 26 juillet 2001). Harcèlement sexuel et droits de la personne [En ligne], Adresse URL: http:// www.ohrc.on.ca/french/guides/sexual-harassment.html

DAVIAU, A. et D. ROLLER (2000). Sommaire et rapport du sondage auprès des membres du personnel (1999), Ottawa, Université d'Ottawa, Bureau d'intervention en matière de harcèlement sexuel.

DE BELLEFEUILLE,J. (1993). Le harcèlement sexuel: non, c'est non!, Montréal, Les Éditions du Remueménage.

DEBOUT, M. et C. LAROSE (2003). Violences au travail: agressions, harcèlements, plans sociaux, Paris, Les Éditions de l'Atelier/Les Éditions ouvrières.

DEJOURS, C. (1988). Plaisir et souffrance dans le travail, 2 volumes, Paris, Édition de l'AOCIP.

GAUVIN, M. (1991). «Le harcèlement sexuel et sexiste comme pratique d'appropriation des femmes: la situation dans les universités canadiennes», Égalité, printemps, 191-233.

GARCEAU, MARIE-LUCE (2002). Le harcèlement sexiste et sexuel en milieu francophone d'éducation postsecondaire dans le Nord de l'Ontario. Rapport d'enquête, Sudbury, Comité ad hoc sur la violence en milieu postsecondaire dans le Nord de l'Ontario.

GAUVIN, M., K. MARCOCCIO et A. GUÉRETTE-BREAU (1999). Le harcèlement sexiste, le harcèlement sexuel et l'agression sexuelle à l'Université de Moncton. Rapport du sondage mené auprès des étudiantes et des employées au Centre universitaire de Moncton, Moncton, Université de Moncton, Groupe de recherche et d'intervention sur le harcèlement sexuel et sexiste en milieu d'enseignement francophone.

HIRIGOYEN, M.-F. (2001). Malaise dans le travail. Harcèlement moral: démêler le vrai du faux, Paris, La Découverte et Syros.

HIRIGOYEN, M.-F. (1998). Le harcèlement moral: la violence perverse au quotidien, Paris, La Découverte et Syros.

LEYMANN, H. (1996). Mobbing, Paris, Seuil.

PALYS, T. (1997). Research Decisions: Quantitative and Qualitative Perspectives, Toronto, Harcourt Brace and Company.

SAVOIE, D. et V. LAROUCHE. (1988). «Le harcèlement sexuel au travail : définition et mesure du phénomène», Relations industrielles, vol. 43, no 2, 509-528.

SOARES,Angelo (2002). Quand le travail devient indécent: le harcèlement psychologique au travail, Montréal, Université du Québec à Montréal, École des sciences de la gestion.

\section{Notes}

1. L'auteure tient à remercier toutes les personnes qui ont œuvré au sein du Comité ad hoc sur la violence en milieu postsecondaire dans le Nord de l'Ontario et, plus particulièrement, Gabrielle Lavigne pour ses commentaires sur ce texte.

2. Le projet «Violence en milieu postsecondaire dans le Nord de l'Ontario: un virage vers l'égalité des femmes» a été financé principalement par Condition féminine Canada, Programme de promotion de la femme. 
3. Il faut noter que le harcèlement sexuel et les remarques ou conduites inconvenantes liées au sexe sont interdites, quel que soit le sexe des personnes concernées. La Commission ontarienne des droits de la personne (2001) a reçu des plaintes portées par des hommes contre des femmes, de même que des plaintes impliquant des personnes de même sexe.

4. Voir, Cantin, S. et C. Proulx (1995); Gauvin, M., K. Marcoccio et A. Guérette-Breau (1999) et, Daviau, A. et D. Roller (2000).

5. Dans cet article, nous ne discuterons pas des résultats des groupes de discussion. Par contre, soulignons qu'ils ont été utilisés plus particulièrement pour cerner les besoins en matière de prévention, d'éducation et de services à mettre en place (Garceau 2002).

6. Le questionnaire utilisé a été construit à partir de trois autres questionnaires utilisés à l'Université de Moncton (Gauvin, Marcoccio et Guérette-Breau 1999) ${ }^{6}$, à l'Université d'Ottawa (Daviau et Roller 2000) et à l'Université de Montréal (Cantin et Proulx 1995).

7. Pour de plus amples renseignements sur le sondage, on peut consulter le document suivant: Garceau, Marie-Luce. 2002. Le harcèlement sexiste et sexuel en milieu francophone d'éducation postsecondaire dans le Nord de l'Ontario. Rapport d'enquête. Sudbury, Comité ad hoc sur la violence en milieu postsecondaire dans le Nord de l'Ontario.

Les données recueillies par le questionnaire ont été traitées à l'aide du logiciel SPSS.

8. Au sujet du taux de réponses, Palys (1997) indique que les envois postaux obtiennent des taux de réponse allant de 10 à $40 \%$ (traduction libre).

9. Dans la présente enquête, le taux de réponse est plus faible que les taux obtenus dans des sondages similaires menés auprès des étudiantes et du personnel des universités de Moncton (entre $24,6 \%$ et $44,1 \%$ ), d'Ottawa (entre $29,8 \%$ et $36 \%$ ) ou de Montréal (entre 37,3\% et $55,2 \%)$.

10. Le nombre de répondantes est de 537.

11. Les pourcentages présentés dans chacune des quatre grandes catégories (climat sexiste, climat empoisonné, harcèlement sexuel et agression sexuelle) sont calculés à partir du nombre de réponses des répondantes - ces réponses sont multiples. Les pourcentages présentés pour chacun des comportements sont calculés à partir du nombre de réponses des répondantes - ces réponses sont multiples.

12. Nous avons classé ces comportements dans la catégorie du harcèlement sexuel à cause de leur contenu pornographique offensant.

13. Seuls les comportements vécus dans les 12 mois qui ont précédé le sondage ont été retenus pour l'analyse des tendances relationnelles.

14. Voir Garceau 2002.

15. Les répondantes invoquent prendre le problème en main parce qu'elles croient que la situation n'est pas suffisamment sérieuse pour porter plainte, qu'elles veulent la régler par elles-mêmes, qu'elles pensent que c'est un problème personnel (Garceau 2002: 39).

16. Voir Garceau 2002. 